Este artículo explora las aportaciones de

DOSSIER $\cdot 95-105$

la traductología teatral al debate sobre la

representabilidad de la última década. Se recogen las delimitaciones al concepto a partir de enfoques textuales, que se circunscriben en prácticas teatrales e ideológicas concretas. Se examina el poder relativo del traductor frente al dramaturgo y la dirección escénica y la negociabilidad de la traducción. Se retoma el debate sobre las traducciones literales y sus adaptaciones por dramaturgos reconocidos, vistas como un tipo de traducción colaborativa y, por último se examina la (ir)representabilidad de traducciones en contextos culturales en conflicto.

Palabras clave: Traducción teatral, representabilidad, oralidad, negociabilidad, traducción colaborativa.

\title{
Repensar la representabilidad
}

This paper explores the contributions of theatre translation studies to the debate on performability in the last ten years. The concept of performability is revised taking into account textual practices, which are inscribed in specific stage practices and ideological issues. The relative role of the translator versus the playwright and stage director is examined, as well as the negotiability of the translation process. The debate on literal translations and their adaptation by famous playwrights is regarded as one type of collaborative translation embedded in a cultural context and in the light of the participants' views. Finally, the (un) performability of translations in cultural contexts in conflict is considered.

KEYWORDS: Drama translation, performability, orality, negotiability, collaborative translation.

Eva ESPASA

Universitat de Vic 
No sé si Susan Bassnett sabía los ríos de tinta que generarían sus escritos sobre la representabilidad (performability), escritos sugerentes, polémicos y con posturas contradictorias al cabo de los años, en los que primero defendía acérrimamente la representabilidad, para luego atacarla de modo frontal (1978, I985, I988, I99I, 1994, I998). Seguramente era perfectamente consciente de su gesto. En traducción teatral siempre se ha hablado, de modo más o menos explícito, de lo que se considera representable y lo que no. Pero el gesto - teatral- de situar la representabilidad justo en el centro del escenario, como protagonista del debate y, sobre todo, de hacer que esta protagonista cambiase de identidad a lo largo de distintas reposiciones, contribuyó a enriquecer el discurso sobre la representabilidad. Los escritos de Bassnett, con títulos tan elocuentes como «The Case Against Performability» (I99I), sirvieron para generar respuestas sobre un debate que, aunque pueda parecer estéril, siempre reaparece en cualquier replanteamiento sobre la traducción teatral, tanto desde la práctica profesional como desde foros académicos.

En este artículo me propongo glosar brevemente el tratamiento que ha recibido la representabilidad en los escritos académicos sobre traducción teatral de los últimos diez años, especialmente los que tratan la representabilidad de modo explícito, y teniendo en cuenta la bibliografía en inglés y español.

El límite temporal es por necesidad simbólica de acotar con una cifra redonda, y también porque su inicio coincide con la celebración del congreso True to Form: On Stage Translation, en septiembre de 1997, Universidad de Hull, Reino Unido, fundamental para la disciplina, y en cuyo seno se debatieron ampliamente cuestiones relacionadas con la representabilidad, muchas de las cuales encontraron reflejo en la publica-

ción Moving Target (Upton, 2000).

En un escrito anterior, me proponía repasar los distintos enfoques desde los que se suele abordar la representabilidad (Espasa, 2000). Apuntaba que en muchos casos se habla de speakability para referirse a la peculiar oralidad del texto dramático, a la necesidad de producir un texto que favorezca la comprensión inmediata y que, en principio, sea fácil de pronunciar. Se trata de decisiones textuales que no se pueden separar de una perspectiva más global, que depende de la orientación teatral de la traducción o de su producción. Es en esos casos cuando se suele hablar de la playability de la traducción. Pero, a su vez, dichas perspectivas no pueden desligarse de cuestiones externas a la producción del texto traducido: cuestiones ideológicas, políticas, o de estatus y prestigio en el mundo teatral. A sabiendas de que dicha cuestión no se reduce a cuestiones comerciales o saleability, esta palabra me servía para comentar el rechazo de Bassnett, desde i99I, del concepto de representabilidad, a partir de prácticas teatrales concretas: la adaptación, por parte de dramaturgos nativos, de obras traducidas «literalmente» por otras personas, que reciben menor remuneración y prestigio (volveremos a esta práctica más adelante). En definitiva, lo que me proponía era incluir cuestiones como la ideología y cuestiones del poder dentro de la noción de representabilidad.

Los escritos de esta última década insisten en la paradójica dificultad y necesidad de abordar la representabilidad. Así, John Sanderson (2002) plantea la paradoja de que, aunque falta una definición precisa y un desarrollo teórico sobre la representabilidad, dicha noción sigue siendo imprescindible para el análisis traductológico. Marta Mateo (2002) analiza de modo muy exhaustivo la representabilidad, incluyendo las relaciones de poder implicadas. Del mismo 
modo, David Johnston (2004) considera que la importancia de la representabilidad para la traducción teatral asegura que seguirá siendo un tema de debate. Johnston concede que sí se ha usado la representabilidad como estrategia de marketing, pero no está de acuerdo con la visión de que sea algo elusivo, incuantificable. E1 nexo de unión de los escritos de la representabilidad en el umbral del milenio es que el debate ya no se centra en si es posible un análisis desde la representabilidad sino en cómo se articula la representabilidad. Y se of recen matizaciones sobre cuestiones que se planteaban anteriormente.

Pilar Ezpeleta apunta, entre otras, tres cuestiones relevantes para el estudio de la representabilidad (2007: 159-160):

I. la necesidad de delimitar los conceptos de teatralidad y representabilidad;

2. la necesidad de perfilar el papel y la responsabilidad del traductor; $y$

3. la consideración de las traducciones como productos culturales en conflicto.

Recogemos el guante y las utilizamos como epígrafes de este repaso sobre la representabilidad.

\section{DELIMITACIÓN DE LOS CONCEPTOS DE TEATRALIDAD Y REPRESENTABILIDAD}

Hablaremos de representabilidad, más que de teatralidad, para evitar entrar en cuestiones sobre la esencia del teatro y la teatralidad. Hablar de representabilidad, en cambio, dirige nuestra atención hacia el escenario de la representación, al examen de qué es lo que hace que una traducción sea representable. Empezaremos por la oralidad del texto traducido.

Para David Johnston, en la traducción, desde la perspectiva de lo que es pronunciable, se debe procurar el equilibrio entre la oralidad
- cotidiana - y extrañeza de los textos teatrales (2004: 35). Así, como nos recuerda Sirkku Aaltonen, no hay que confundir la oralidad con la simplicidad. Los textos teatrales no tienen por qué ser sencillos ni fáciles de pronunciar (Aaltonen, 2000: 42-43; véase también Sanderson, 2002: 49). Asimismo David Johnston, aunque defiende la pericia lingüística del traductor, que hace que el texto sea pronunciable y favorezca la comprensión por parte del público, nos alerta del riesgo de caer en la «banalidad», citando a Patrice Pavis (1989: 30), quien advierte que no hay que confundir un texto pronunciable con una norma de verosimilitud teatral.

La tensión entre traducción pronunciable y texto teatral (más o menos) verosímil es una muestra de que los criterios textuales dependen de una concepción dramática general de la obra. En este sentido, y como nos recuerda David Johnston, el traductor debe ubicar en el diálogo los puntos en que las pautas conversacionales se combinan con marcas significativas en el discurso de la obra. Considera que el problema más habitual de las traducciones representadas es la excesiva normalización del texto: «el lenguaje que no resulta problemático de algún modo no es materia prima del teatro» (2004: $35)^{\text {r. }}$ Pero también puede haber el riesgo de «tocar notas falsas» y enfatizar elementos puntuales que pueden resultar contraproducentes para el desarrollo de la obra. La solución, para Johnston, pasa por la asistencia del traductor a los ensayos. La lectura en voz alta no es suficiente: el significado teatral se produce en los intercambios entre personajes, $y$ no en las intervenciones individuales. En este sentido cabe recordar las aportaciones de Raquel Merino y su defensa de la réplica como unidad estructural mínima del campo dramático, por contener

I Las traducciones de las citas son mías.

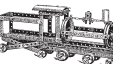


diálogo y también marco (como por ejemplo, acotaciones) (Merino, 1994: 44- 46).

El sólido estudio de John Sanderson (2002) sobre las pautas fónicas para la traducción teatral de obras de Shakespeare, incide en la necesidad de contemplar cuestiones como la longitud de la traducción, su ritmo subyacente, la carga semántica de efectos sonoros recurrentes, así como la autonomía del texto traducido: aunque los elementos kinésicos y escenográficos puedan compensar posibles aspectos que requieran aclaración, la traducción - previa a la representación- no tiene por qué contar con esta posibilidad y Sanderson propone que se aporten soluciones intratextuales. Aunque se puedan incluir notas a pie de página en traducciones publicadas para explicar los ajustes producidos en la traducción, el texto debería ser lo suficientemente autónomo para la lectura y la potencial representación (2002: 45-53). Sanderson considera que la atención a los recursos fónicos no tiene por qué ser exclusiva de la traducción escénica, aunque sí suela ser prioritaria en esta. Paralelamente, a pesar de la posible percepción actual de los recursos retóricos como formas lingüísticas obsoletas, Sanderson examina cómo las traducciones recientes presentan más figuras de dicción que traducciones más antiguas (2002: 357). Se corrobora así que los recursos textuales no se contraponen a criterios teatrales: al contrario, están al servicio de estos.

\section{Sobre la prioridad escrita o escénica de la traducción}

Ekaterini Nikolarea (2002) ofrece un repaso histórico de la representabilidad en traducción teatral, a partir de la construcción de un debate entre legibilidad y representabilidad (readability/performability). Tras un completo resumen de las aportaciones a la semiótica teatral de la

Escuela de Praga, así como de Tadeusz Kowzan y de Anne Ubersfeld (que también recogen, por ejemplo, Aaltonen, 2000 y Ezpeleta, 2007), Nikolarea repasa los escritos de Susan Bassnett y de Patrice Pavis sobre la representabilidad de la traducción teatral. Resigue con detalle los argumentos de Bassnett, quien primero defendía enfáticamente la adscripción de la traducción dentro de la representación, para concluir, en 1991, que las únicas vías de investigación válidas en traductología teatral serían: I) una historiografía de la traducción teatral o 2) más investigación en las estructuras lingüísticas de los textos teatrales existentes. Según Nikolarea, las diferencias entre las posturas de Pavis (pro representabilidad) y Bassnett (pro legibilidad), responden a la adscripción de los autores a distintas escuelas de investigación. Pavis, procedente de la semiótica teatral, se interesó por la traducción teatral como proceso, y por la «universalización» de la cultura (I989: 42). En cambio, para Nikolarea, el hecho de que Susan Bassnett formase parte de la Escuela de la Manipulación, le llevaba a centrarse en la traducción como producto, dentro de la cultura de llegada. Este punto es discutible, ya que la llamada Escuela de la Manipulación, como los estudios descriptivos de la traducción, se centra tanto en el producto como también en el proceso y en la función de los textos traducidos. Lo que sí es cierto es que, como bien apunta Nikokarea, el interés de Bassnett por la antropología teatral le llevaría a observar la especificidad de la cultura más que su supuesta universalidad. De hecho, Pavis ha recibido críticas por sus visiones universalistas de la cultura (véase Cameron, 2000), frente al respeto generalizado de su visión sobre traducción, profundamente anclada en el teatro. Lo que me interesa observar en este punto es cómo el sólido repaso teórico de semiótica teatral que ofrece Nikolarea 
no le resulta suficiente para explicar las visiones cambiantes de Bassnett y su oposición a Pavis (en el debate construido por Nikolarea). Es la adscripción ideológica e institucional la que explicaría dichas divergencias. Volveremos a cuestiones ideológicas más adelante.

Aaltonen analiza el debate sobre la representabilidad enmarcándolo en el debate más general entre la (supuesta) fidelidad y la libertad de la traducción, y su respectiva pertenencia a los sistemas literarios o teatrales. Los requisitos de oralidad y representabilidad, presentes en los textos teatrales, los separan de la perspectiva dominante en el sistema literario, sobre la relación entre las traducciones y sus textos de partida. Si las normas favorecen una traducción «fiel», a partir de las leyes de propiedad intelectual, ¿cómo se justifican las traducciones más «libres»? En el caso del teatro, se justificarían por los requisitos escénicos de representabilidad (2000: 43).

Para David Johnston, tanto las traducciones publicadas como las escénicas tienen un elemento en común: la preocupación por su recepción. Si la escritura dramática ya contempla un público potencial, su traducción también: ambas suponen viajes hacia la otredad. Pero, de hecho, Johnston muestra su prioridad escénica al entrar más a fondo en las traducciones filológicas. Da el ejemplo de Valle-Inclán, cuyas traducciones filológicas pueden contribuir a dar a conocer su teatro en un público de habla inglesa. Pero quien haya traducido sólo el texto verbal, con una panoplia de notas a pie de página, debe mostrar disposición a que una versión sustituya o se desarrolle a partir de un texto que se presenta sobre todo como un texto para la leer (2004: 27). O sea, aunque en teoría se pueda escribir una traducción que tanto sirva para la página como para la escena, en realidad considera que hay pocas. Y que escribir para la representación ubica al traductor como escritor, involucrado en la práctica interactiva de la creación teatral. Considera que el hecho de centrarse en la producción supone ubicar el debate de la traducción teatral en el contexto de una lucha creativa (Johnston, 2004: 25), especialmente en el proceso del trabajo de mesa y de los ensayos.

Todo ello le lleva a considerar el traductor como representante del autor en la lengua de llegada. En este sentido cabe considerar: I) qué papel tiene el dramaturgo en los ensayos y 2) si existe una base para conceder un rol distinto al traductor como dramaturgo. Repasa brevemente el papel canónico — cambiante a lo largo de la historia - del dramaturgo, sacralizado en el romanticismo, pero con un rol más colaborativo antes y después, sobre todo en el siglo xx, cuando el teatro se alejó del dominio autorial para favorecer procesos más colaborativos. O sea, el rol del dramaturgo ha evolucionado y tiene en cuenta tanto el trabajo colaborativo de los ensayos como la tarea individual de la escritura. Con todo, el estatus de la traducción es algo distinto del de la escritura original: «Prácticamente por definición, una traducción es un artículo intrínsecamente más negociable que un escrito original, esencialmente porque la traducción es, de por sí, el resultado final de un complejo proceso de negociación a partir de la lengua de partida» (2004: 3I).

Johnston proporciona un ejemplo propio de la provisionalidad y negociabilidad percibida del texto: la práctica, habitual entre directores y actores, de traer a los ensayos otras versiones de la obra. Aunque concede que el propósito principal es el de profundizar en las complejidades de la obra, dicha práctica también puede cuestionar el estatus canónico del texto traducido (2004: 32).

Johnston también matiza los límites de la negociabilidad del texto teatral traducido: la 
multiplicidad de interpretaciones no implica que exista una gama infinita de significados en un texto dado. Normalmente el escritor, o el 100 traductor como escritor, tiene el conocimiento y el contexto que le autorizan para juzgar las relaciones entre escritura y representación: «Los traductores no asisten o participan en los ensayos para salvaguardar la pureza de las intenciones del autor original [...]. Lo que buscan es asegurar que las decisiones de la producción y las percepciones artísticas se ubiquen en las posibilidades contextuales de la obra» (2004: 33).

En definitiva, observamos que Johnson sí se decanta por las traducciones escénicas y, sobre todo, por la interlocución necesaria del traductor, respecto a otros agentes de la comunicación teatral: «en una obra teatral traducida, nadie tiene mejor ubicación [...] que el traductor para renegociar el texto para el actor, para así asegurar su representabilidad» (2004: 37). Aunque concede que el traductor no posee conocimiento directo de estas dimensiones cruciales, y que se debe buscar un equilibrio atento entre licencias excesivas de interpretación y una cerrazón mortal (deadening closure) (2004: 33).

Respecto a la dualidad del texto dramático (escrito/representado), Marta Mateo desmonta los términos habituales del debate cuando nos recuerda que la representación es precisamente una de las posibles interpretaciones del texto escrito: «la posibilidad de distintas interpretaciones tanto del texto escrito como del oral tiene indudables consecuencias para la traducción, que a su vez se puede concebir como otra posible interpretación más del texto dramático de origen» (2000).

$\mathrm{Y}$, de modo más general, en su completo estudio sobre la representabilidad, entendida como estrategia global de traducción teatral, Marta Mateo enumera los elementos que tendría que contemplar (2000):
I. la posibilidad de la representación;

2. la fuerza de la palabra oral;

3. la importancia de la presencia del receptor en el acto comunicativo de la puesta en escena; y

4. la economía semiótica de la obra dramática (con la posibilidad de intercambio de distintos códigos semióticos).

Todos estos aspectos se deberían observar desde la teoría de la relevancia, según la cual en la traducción se busca el máximo efecto con un mínimo esfuerzo, sin obviar las restricciones de la traducción teatral y explotando las posibilidades semióticas del proceso.

En su modelo de análisis de los textos dramáticos para la traducción, Ezpeleta delimita cuestiones pertinentes, como por ejemplo la distinción, desde la perspectiva comunicativa, entre la comunicación externa (entre actores y público) y la comunicación interna (entre personajes, dentro de la ficción dramática) (2007: 384-386). Realmente, ningún estudio sobre la representabilidad puede pasar por alto estas cuestiones sin correr el riesgo de banalizar la oralidad, como ya hemos apuntado más arriba, como si se tratase sólo de la comprensión inmediata por parte del público (o de los actores), y no de una ficción dramática en que la oralidad muestra gran estilización, con propósitos dramáticos que no tienen por qué ser realistas.

Un modo de poner a prueba en qué consiste la representabilidad es examinar obras que se han retraducido para la escena y observar su contexto y estrategias traductoras utilizadas. En este sentido, Sirkku Aaltonen (2003) ha estudiado distintos contextos de retraducción en el teatro finés:

I. Traducciones, normalmente escritas para publicación, que se retraducen cuando cambia el canon estético, como obras 
traducidas en el siglo XviII, en las que se adaptan los referentes culturales. Con los nuevos cánones del romanticismo, se favorecen retraducciones más extranjerizantes que busquen un mayor acercamiento a la obra, autoría y culturas de partida.

2. En el contexto de exportación de obras teatrales finlandeses, las traducciones (dirigidas a dramaturgos de la cultura receptora) se retraducen para producciones concretas.

3. Retraducciones en la escena finesa contemporáneas, de acuerdo con las preferencias escénicas de la producción, cuando se quiere resaltar una lectura concreta de la obra, una estrategia de traducción, o cuando se considera obsoleto el lenguaje

(Aaltonen, 2003: 154, 156).

\section{EL PAPEL Y LA RESPONSABILIDAD DEL/LA TRADUCTOR/A}

Ya hemos visto cómo David Johnston defiende el rol del traductor para asegurar la representabilidad del texto, en un artículo titulado significativamente «Securing the Performability of the Play in Translation» (2004). Dicho rol no siempre es reconocido así. Por una parte, suele recaer en la dirección escénica la toma última de decisiones (aunque se trate de un proceso colaborativo). Por otra parte, en el teatro representado en inglés es una práctica habitual el hecho de encargar traducciones «literales» hacia el inglés, que después «adaptan» dramaturgos de prestigio: son estos quienes constan como «adaptadores» en la promoción de la obra, y quienes son mejor remunerados. La autoría de las traducciones literales queda relegada a un segundo plano o al anonimato. El hecho de que dichos textos en inglés se denominen literals como sustantivo, ya no como adjetivo, nos muestra que se trata de un concepto en sí. Desde la traductología contemporánea se cuestiona la posibilidad de la traducción literal, porque toda traducción conlleva interpretación: «la neutralidad y falta de creatividad de la traducción literal es cuestionable, porque cualquier reescritura contiene una fase de interpretación» (Perteghella, 2004: 15). Pero dicha práctica traslativa existe, establece roles diferenciados y tiene consecuencias para el reconocimiento de la autoría de la traducción.

Podemos considerar esta práctica como síntoma del papel periférico de la traducción en el mundo teatral de habla inglesa: para que se considere representable un texto tiene que invisibilizarse su estatus como traducción, y visibilizarse su adscripción al sistema teatral, mediante la firma de un dramaturgo. En definitiva, el estatus de la traducción se considera inferior al de la escritura teatral mediante el criterio de la representabilidad. Y quizá fue este uno de los motivos por los que Susan Bassnett inició su cruzada contra la representabilidad (véase Bassnett, 199r; Espasa, 2000).

Mateo (2002: 54-55) apunta argumentos muy pertinentes al debate. En primer lugar, la consideración negativa de la representabilidad puede proceder del constante recurso a dicha noción para justificar la práctica de traducción literal/ adaptación con dramaturgo de renombre. En la polémica fallan dos aspectos: los partidarios de esta práctica de traducción parecen negar la posibilidad de que un traductor pueda dotar de representabilidad a sus textos. Además, la noción de representabilidad no tiene por qué vincularse necesariamente con dicha práctica, como hace Bassnett (Mateo, 2002: 55): aunque en los últimos escritos Bassnett se muestra reacia a la noción de representabilidad, sí la 
tiene en cuenta en su práctica teatral. En una traducción para BBC Radio, realizada junto a David Hirst, de Trovarsi de Pirandello, 102 Bassnett describe las estrategias tomadas: adición de los nombres de personajes, para facilitar el seguimiento radiofónico; adaptación de la duración del texto, de acuerdo con el tiempo de transmisión. Para Mateo, estas estrategias se pueden interpretar como parte de un concepto de representabilidad.

Phyllis Zatlin es autora de un interesantísimo estudio que recoge su propia perspectiva como traductora teatral, así como la voz de otros traductores, a partir de sus respuestas a un cuestionario elaborado por la autora (2005). Respecto a las adaptaciones de dramaturgos reconocidos, recoge la queja de que, entre la plantilla de traductores anglófonos de Chejov figuran dramaturgos de primer orden, que desconocen el ruso (con la excepción de Michael Frayn). Considera que sería mejor encargar nuevas traducciones de obras desconocidas, en vez de más retraducciones de obras conocidas (Boehm en Zatlin, 2005: 35).

Zatlin también da un ejemplo de restricciones legales a la representabilidad: una traducción existente de una obra contemporánea rusa se convirtió, a efectos prácticos, en irrepresentable cuando el dramaturgo ruso Aleksandr Buravsky firmó un pacto de exclusividad con la compañía teatral estadounidense, en el que se especificaba su preferencia por una versión a cargo de un dramaturgo estadounidense, a partir de una versión «literal» de una persona nativa rusa (op. cit.: 26). Pero Zatlin también dedica un capítulo a traducciones colaborativas que sí funcionan, especialmente cuando se dan en redes de colaboración con relaciones más equitativas entre sus miembros (op. cit.: 53-66).

Manuela Perteghella ha investigado a fondo la cuestión de las traducciones colaborativas y

ha determinado, dentro de un modelo general de traducción teatral, distintas tipologías, de las que destacamos las que implican distintos grados de colaboración textual o personal (2004: II-I6):

I. traducción colaborativa, con intercambios entre traductor y dramaturgo, a menudo con la mediación de agente literario del dramaturgo;

2. teatro patchwork o collage. Uso deliberado de distintas traducciones yuxtapuestas, práctica que se asocia a producciones postmodernas;

3. adaptación, de la mano de dramaturgo reconocido, a partir de una traducción literal (a menudo acompañada de notas, en el que el o la traductora funciona de fidus interpretes del texto de partida). Ya hemos comentado dicha práctica, pero Perteghella distingue dentro de esta las siguientes fases:

a. traducción filológica: la prioridad es la exactitud textual, por encima de convenciones teatrales. Se corresponde con la versión literal; y

b. adaptación lingüística o cultural, por parte de dramaturgo de prestigio. Suele conllevar la sustitución de referentes lingüísticos o culturales.

Lo que es especialmente interesante del estudio de Perteghella es que, en primer lugar, enmarca distintas formas de traducción colaborativa. Aunque se muestra crítica respecto a la posibilidad de elaborar una traducción meramente literal, su estudio no se estanca en ello, y describe distintas prácticas traductoras, dentro de un modelo general de traducción teatral. También es muestra de los escritos que más que prescribir sobre una práctica concreta, lo que hacen es describir los contextos en los que se generan. Así, Sirkku Aaltonen también 
comenta que el encargo de una traducción literal se justificaría cuando se necesita un puente entre idiomas de menor difusión y la escena receptora; la justificación radicaría en posibilitar intercambios teatrales interculturales (2003: I52). No se justificaría en cambio, para retribuir menos a quien realice la traducción literal en beneficio de un dramaturgo conocido (2000: 44 y 2003: 152 ).

Marta Mateo (2000) comenta dicha práctica de traducción recogiendo las opiniones de participantes en el sistema, en la cultura británica. $\mathrm{Y}$ nos recuerda que las prácticas traductoras vienen determinadas por las normas de cada polisistema. En una línea afín, Bill Findlay, traductor al escocés de Goldoni a partir de una traducción literal, justifica su motivación (2006): en la escena británica es una práctica reconocida la inclusión del «traductor como escritor» o, quizás con más exactitud, del «escritor como traductor» (ib.: 46). Asimismo, defiende la práctica, no estándar, de traducir de dialecto a dialecto, y se muestra más capacitado para escribir en escocés que para traducir de dialectos italianos. Y esto nos lleva al último punto, de la traducción como producto intercultural, pero desde la visión de la cultura como sede de conflicto, más que de consenso.

\section{TRADUCCIONES COMO PRODUCTOS CULTURALES EN CONFLICTO}

Los intercambios culturales no son inocentes y, como nos recuerda Sirkku Aaltonen (2000), toda traducción es una muestra de egotismo: parte de la propia cultura, que busca reflejarse en otras. El teatro intercultural también se ve como expropiación cultural: así denomina Derrick Cameron los conflictivos intercambios multiculturales y multirraciales en el Reino Unido en el nuevo milenio (2000). Ya no se habla sólo de traducción sino de tradaptación, término usado en el discurso intercultural o postcolonial, para indicar una nueva reescritura, desde una perspectiva no occidental (en Perteghella 2005: I5-18).

Joseph Che Suh, en su tesis doctoral sobre el autor camerunés Oyono Mbia, se propone examinar las nociones de representabilidad del teatro bulu, y cómo se plasman en las obras que el dramaturgo escribe en francés y después autotraduce al inglés (Suh, 2005). Che Suh nos recuerda que la perspectiva de la reculturación no debe olvidar la introducción de teatro como elemento "exótico», como documento de una cultura extranjera, sin pretensión de integrar dicha cultura en el sistema de llegada (ib.: 88). Así, las obras teatrales africanas, incluso las escritas en idiomas europeos (francés o inglés) o traducidas a estos, no se integran en los sistemas culturales europeos, sino africanos, por lo tanto se deben evaluar según estos estándares, de representabilidad. De este modo, las autotraducciones del autor Oyono Mbia son expresamente extranjerizantes, para reflejar la africanidad de los textos, concretamente en la cultura bulu. También considera Suh que este tipo de representabilidad es coherente con la tendencia general de africa nizar idiomas coloniales, y es muestra también del rol ambiguo del escritor/autotraductor por el hecho de escribir en idiomas no africanos (op. cit.: 197). Dicha práctica es afín a las adaptaciones de obras teatrales occidentales realizadas por Wole Soyinka. El dramaturgo nigeriano subvierte la presunta superioridad de los originales clásicos al introducir elementos de las culturas africanas en sus traducciones, como resistencia ante el imperialismo (Lefevere, 1980; 1984).

La representabilidad puede tener una dimensión explícitamente política: ciertas combinaciones lingüísticas de traducción son poco 
representables en contextos de clara tensión política. Un claro ejemplo es la poca traducción y representación en hebreo de obras escritas en 104 árabe, como muestran los estudios de Hannah Amit Kochavi (2000; 2008). Esta investigadora ha examinado la relación entre las políticas de traducción y la evolución del conflicto palestino-israelí. Ha analizado las resistencias que se encuentran para representar obras árabes traducidas al hebreo en Israel, incluso en ambientes supuestamente críticos con las políticas israelíes oficiales, como son los ámbitos teatrales e universitarios. Dicha tensión se traduce en la representación de pocas obras árabes traducidas al hebreo, a su presentación en circuitos teatrales alternativos o universitarios, con pocas representaciones, así como a la utilización de lecturas dramatizadas, de menor repercusión social que las escenificaciones.

Podemos concluir afirmando que el debate sobre la representabilidad se ha afinado. En el umbral del milenio se han reducido las polaridades dicotómicas sobre orientación escrita y orientación escénica de la traducción; se ha profundizado en las distintas prácticas de traducción colaborativa, cambiantes según los contextos teatrales y culturales; y se han abierto más ventanas a expresiones menos eurocéntricas sobre la representabilidad.

RECIBIDO DICEMBRE 2008 ACEPTADO FEBRERO 2009

\section{REFERENCIAS BIBLIOGRÁFICAS}

Aaltonen, S. (200o). Time-Sharing on Stage: Drama Translation in Theatre and Society, Clevedon: Multilingual Matters.

- (2003). «Retranslation in the Finnish Theatre». Cadernos de Traduçao, II, pp. I4I-I59.

Amit-Kochavi, H. (2000). «Hebrew Translations of Palestinian Literature - from Total Denial to Partial Recognition». TTR : traduction, terminologie, rédaction, $\mathrm{I} 3 / \mathrm{I}, \mathrm{pp} .53-8 \mathrm{o}$.

(2008). «Arabic plays translated for the Israeli Hebrew Stage - a descriptive-analytical case study», en A. Pym, M. Shlesinger y D. Simeoni (eds.) Beyond Descriptive Translation Studies. Investigations in homage to Gideon Toury, Amsterdam: John Benjamins.

Bassnett, S. (1978). «Translating Spatial Poetry: An Examination of Theatre Texts in Performance», en J. S. Holmes, J. Lambert y R. van den Broeck (eds.) Literature and Translation. New Perspectives in Literary Studies, Lovaina: Acco, pp. I6I-I76. - (1985). "Ways Through the Labyrinth: Strategies and Methods for Translating Theatre Texts», en T. Hermans (ed.) The Manipulation of Literature: Studies in Literary Translation, Londres: Croom Helm, pp. 87- 102.

- (1988). Translation Studies, Londres: Routledge, I980.

- (I99I). «Translating for the Theatre: The Case Against Performability». TTR : traduction, terminologie, rédaction, $4 / \mathrm{I}$, pp. 99-III.

_- (I994). Translation Studies, edición revisada, Londres: Routledge, I991.

_ (I998). «Still Trapped in the Labyrinth: Further Reflections on Translation and Theatre», en S. Bassnett y A. Lefevere (eds.) Constructing Cultures: Essays on Literary Translation, Clevedon: Multilingual Matters, pp. 90-Io8.

Cameron, D. (2000). "Tradaptation: Cultural Exchange and Black British Theatre», en C. A. Upton (ed.), pp. I7-24.

Coelsch-Foisner, S. y H. Klein (eds.) (2004). Drama Translation and Theatre Practice, Frankfurt: Peter Lang.

Ezpeleta, P. (2007). Teatro y traducción. Aproximación interdisciplinaria desde la obra de Shakespeare, Madrid: Cátedra.

Espasa, E. (2000). «Performability in Translation: Speakability? Playability? Or just Saleability?», en C. A. Upton (ed.), pp. 49-62.

(200I). La traducció dalt de l'escenari, Vic: Eumo. (2004). "Theatre and Translation. Unequal Exchanges in a Supermarket of Cultures», en A. Branchadell y M. L. West (eds.) Less Translated Languages, Amsterdam: John Benjamins.

Findlay, B. (2006). «Motivation in a Surrogate Trans- 
lation of Goldoni», en S. Bassnett y P. Bush (eds.) The Translator as Writer, Londres: Continuum International Publishing Group, pp. 46-57.

Johnston, D. (2004). «Securing the Performability of the Play in Translation», en S. Coelsch-Foisner y H. Klein (eds.), pp. 25-38.

Lefevere, A. (I980). "Translation: Changing the Code; Soyinka's Ironic Aetiology», en O. Zuber (ed.) The Languages of Theatre: Problems in the Translation and Transposition of Drama, Oxford: Pergamon.

Lefevere, A. (1984). «Refraction: Some Observations on the Occasion of Wole Soyinka's Opera Wonyosi», en O. Zuber (ed.) Page to Stage: Theatre as Translation, Amsterdam: Rodopi, pp. I9I-I98.

Mateo, M. (2000). «La 'representabilidad' como eje de las decisiones y discusiones sobre la traducción teatral». Ponencia plenaria presentada en les $I V$ Jornades de Traducció a Vic: La traducció teatral, Vic: Universitat de Vic, 6-7 abril 2000.

- (2002). «Power Relations in Drama Translation», en I. Dimitriu, (ed.) 'Translation, Diversity and Power', monográfico de Current Writing. Text and Reception in Southern Africa, I4/2, pp. 45-63.

Merino, R. (1994). Traducción, tradición y manipulación: teatro inglés en España (1950-I990), León: Servicio de Publicaciones del País Vasco.

Nikolarea, E. (2002). «Performability versus Read- ability: A Historical Overview of a Theoretical Polarization in Theater Translation». Translation Journal, 6/4. <http://accurapid.com/ journal/22theater.htm $>$ [Consulta: 30 octubre 2008].

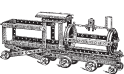

Pavis, P. (I989). «Problems of Translation for the Stage: Interculturalism and Post-Modern Theatre», en H. Scolnicov y P. Holland (eds.) The Play Out of Context: transferring Plays from Culture to Culture, Cambridge: Cambridge University Press, traducción inglesa del artículo de L. Kruger, pp. 25-44.

Perteghella, M. (2004). «A Descriptive-Anthropological Model of Theatre Translation», en S. Coelsch-Foisner y H. Klein (eds.), pp. I-23.

Sanderson, J. (2002). Traducir el teatro de Shakespeare: figures retóricas iterativas en Ricardo III. Valencia: Universitat de València.

Suh, J. C. (2005). A Study of Translation Strategies in Guillaume Oyono Mbia's Plays. Tesis doctoral, University of South Africa.

Upton, C. A. (ed.) (2000). Moving Target: Theatre Translation and Cultural Relocation, Manchester: Saint Jerôme.

Zatlin, P. (2005). Theatrical Translation and Film Adaptation: A Practitioner's View, Clevedon: Multilingual Matters. 